Centrum voor Wiskunde en Informatica REPORT RAPPORT

Pattern Formation in Electric Discharges

U.M. Ebert, M. Arrayás

Modelling, Analysis and Simulation (MAS)

MAS-R0028 October 31, 2000 
Report MAS-R0028

ISSN 1386-3703

CWI

P.O. Box 94079

1090 GB Amsterdam

The Netherlands

$\mathrm{CWI}$ is the National Research Institute for Mathematics and Computer Science. CWI is part of the Stichting Mathematisch Centrum (SMC), the Dutch foundation for promotion of mathematics and computer science and their applications.

SMC is sponsored by the Netherlands Organization for Scientific Research (NWO). CWI is a member of ERCIM, the European Research Consortium for Informatics and Mathematics.

Copyright @ Stichting Mathematisch Centrum P.O. Box 94079, 1090 GB Amsterdam (NL) Kruislaan 413, 1098 SJ Amsterdam (NL) Telephone +31205929333 Telefax +31205924199 


\title{
Pattern Formation in Electric Discharges
}

\author{
Ute Ebert ${ }^{1}$ and Manuel Arrayás ${ }^{1,2}$ \\ ${ }^{1}$ CWI, P.O. Box 94079, 1090 GB Amsterdam, The Netherlands \\ ${ }^{2}$ Instituut-Lorentz, P.O. Box 9506, 2300 RA Leiden, The Netherlands
}

\begin{abstract}
Electric discharges with low current densities can be described by a minimal model of reaction, drift and diffusion of two charged species coupled nonlinearly to an externally imposed electric field. The intrinsic ionization-fieldcoupling of the model leads to pattern formation both in stationary or periodically driven barrier discharges and in transient streamer discharges. In particular, we discuss negative streamer discharge fingers extending into a non-ionized area. They belong to the class of Laplacian growth phenomena. For quantitative predictions, a systematic derivation of the corrections to the velocity of a moving boundary due to curvature or relaxation effects is still missing. This is because negative streamer fronts are so-called pulled fronts, for which standard approximation schemes fail. We present new approaches and results.
\end{abstract}

2000 Mathematics Subject Classification: 35B05, 35P99, 35Q60

Keywords and Phrases: Pattern formation, electric discharges, barrier discharges, streamers, reaction-driftdiffusion-models, front solutions, Laplacian growth, linear perturbation theory, moving boundary approximation Note: Ute Ebert leads CWI-project MAS 1.4 Pattern formation and low temperature physics. She was financially supported by the Dutch Science Foundation NWO. Manuel Arrayás is supported by the European network "Patterns, noise and chaos". The paper was submitted to a special issue of Lecture Notes in Physics (Springer) on July 26, 2000.

\section{Modelling Electric discharges}

\subsection{Phenomenology, spatio-temporal patterns}

Electric discharges are commonly known from natural phenomena like sparks whose lengths can vary from cm's to km's, or St. Elmo's fire (a corona discharge at the tops of ship masts). Neon tubes are the best known example from a whole list of technical applications that are under continued use and investigation. For an overview of phenomena in gas discharges, we refer to [1]. Discharges can occur not only in gases, but also in fluids or solids - in just any matter that can turn from a state of low or vanishing conductivity to a state of high conductivity, when a sufficiently strong field is applied.

The name "discharge" is a historical one like many other ones in this field, since it refers to the discharge of the voltage stored in a capacitor, when the matter between the capacitor plates becomes conducting. However, a sufficiently strong voltage source also might sustain the voltage difference between the outer electrodes despite the "discharge" in the bridging matter. Electric discharges can create a low temperature plasma locally. In contrast to high temperature plasmas as in fusion reactors or stars that exist due to heating and confinement, low temperature plasmas exist under nonequilibrium conditions due to external excitations like electromagnetic fields. Hence they generically are inhomogeneous in space and time and form spatio-temporal patterns. The homogeneous glow of the neon tube is an exception and a result of the art of the engineer. The onset of spatio-temporal instabilities limits the range of technical applicability in quite a number of cases.

Below, we will focus on streamer and barrier discharges in simple matter like pure nitrogen under normal conditions. Streamers are the initial breakdown mode of insulating matter on length scales from cm's to m's after a voltage shock has been applied. They are extending finger-like patterns of 
ionized matter, and also the precursors of a later short-circuit, if the conducting channel eventually contacts both electrodes. Initially during the streamer phase, the degree of ionization stays relatively low.

Barrier discharges are operated in a stationary or periodically changing electric field. They consist of a layered structure of a discharge with at least one resistive layer in dc fields or at least one dielectric layer in ac fields. High currents and degrees of ionization are then prevented by the dielectric or resistive layers, just as in the case of a large external load resistance in the electric circuit.

\subsection{The minimal model of low current discharges and the ionization-field-coupling}

Pattern formation occurs in nonlinear spatially extended systems under nonequilibrium conditions. The nonequilibrium here is due to an externally applied electric field. The nonlinear mechanism to be explored is an ionization-field-coupling: a sufficiently high electric field leads to a multiplication of charge carriers by a local impact ionization reaction. The generated charges drift in the electric field. If their density is sufficiently high, they will modify the field and hence change the local reaction rates and drift velocities. Accordingly, the evolution of the local degree of ionization is determined by a fully dynamic process. The situation is captured by the following model:

In general, the continuity equations for particles of species $i$ are

$$
\partial_{t} \rho_{i}+\nabla \cdot \mathbf{j}_{i}=f_{i}\left(\left\{\rho_{j}\right\}, \mathbf{E}\right) \quad, \quad \mathbf{j}_{i}=q_{i} \mu_{i} \rho_{i} \mathbf{E}-D_{i} \nabla \rho_{i}
$$

where $\rho_{i}$ is the particle density, $q_{i}= \pm$ the sign of the charge, $\mu_{i}$ the mobility and $D_{i}$ the diffusion constant of species $i$. If the degree of ionization is sufficiently small, dissipative heating can be neglected and the neutral background stays unchanged. The current $\mathbf{j}_{i}$ then can be approximated as in (1.1) by particle diffusion and Ohmic friction. This is true in gases, liquids or solids. In a semiconductor, the current $\mathbf{j}_{i}$ can saturate for large $\mathbf{E}$. $f_{i}$ is the source term describing the reactions of the particles in the local field. If the currents stay sufficiently small, also magnetic and relativistic effects can be neglected, and we only need the Poisson equation of electrostatics

$$
\frac{q}{\varepsilon_{0}}=\sum_{i} \frac{q_{i} e \rho_{i}}{\varepsilon_{0}}=\nabla \cdot \mathbf{E}, \quad \mathbf{E}=-\nabla \Phi,
$$

where $e$ is the electron unit charge and $\epsilon_{0}$ the dielectric constant. The dominant reaction in a strong electric field is impact ionization: a light charged particle, typically an electron $\mathrm{e}^{-}$, can react with a neutral atom or molecule $\mathrm{A}$ as: $\mathrm{e}^{-}+\mathrm{A} \longrightarrow 2 \mathrm{e}^{-}+\mathrm{A}^{+}$, if local electric field times mean free path of the electron are of the order of the ionization energy.

In the simplest case (for a more extended discussion, see [2, 3]), there are only two charged species in a gas, namely electrons (denoted by $i=e$ ) and positive ions $\left(i=+\right.$ ), and the reaction terms $f_{i}$ consist of impact ionization and recombination only:

$$
f_{e}\left(\rho_{e}, \rho_{+}, \mathbf{E}\right)=f_{+}=\left|\mu_{e} \rho_{e} \mathbf{E}\right| \alpha(|\mathbf{E}|)-\beta \rho_{e} \rho_{+} .
$$

Due to charge conservation, the two reaction rates $f_{e}$ and $f_{+}$are identical. The effective cross-section $\alpha(|\mathbf{E}|)$ has approximately a threshold dependence on the electric field. The traditional Townsend approximation [1] reads, e.g.

$$
\alpha(|\mathbf{E}|)_{\text {Townsend }}=\alpha_{0} e^{-E_{0} /|\mathbf{E}|} .
$$

The homogeneous equilibrium conductivity due to the electrons for a given field $\mathbf{E}$ then is $e \mu_{e}^{2}|\mathbf{E}| \alpha(|\mathbf{E}|) / \beta$, and hence the equilibrium current $j(E)$ is proportional to $|\mathbf{E}|^{2} \alpha(|\mathbf{E}|)$. In this model, $j$ is a monotonically increasing function of $E$, so there is no bistability of the current-voltagecharacteristics on the local level of description. Therefore the well-known instability mechanism [4] due to S- or N-shaped current-voltage-characteristics does not apply to simple nitrogen.

If furthermore bulk recombination can be neglected because of a separation of time scales in the streamer discharge or because of the dominance of boundary recombination processes in the barrier 
discharge, and if the mobility of the ions is neglected because $\mu_{+} \ll \mu_{e}$ and $D_{+} \ll D_{e}$, we arrive at the minimal model of low current discharges. In dimensionless form, it reads [5, 2]:

$$
\begin{aligned}
\partial_{t} \sigma-\nabla \cdot(\sigma \mathbf{E}+D \nabla \sigma) & =\sigma f(|\mathbf{E}|), \quad \text { with, e.g. } f(E)=|E| e^{-1 /|E|} \\
\partial_{t} \rho & =\sigma f(|\mathbf{E}|), \\
\nabla \cdot \mathbf{E} & =\rho-\sigma, \quad \mathbf{E}=-\nabla \Phi .
\end{aligned}
$$

$\sigma$ and $\rho$ are the electron and ion particle densities, and $\mathbf{E}$ is the electric field. The dimensionless diffusion constant $D=D_{e} \alpha_{0} /\left(\mu_{e} E_{0}\right)$ is the only intrinsic parameter. For nitrogen under normal conditions the units in (1.5)-(1.7) are [2, 6]: $2 \mu \mathrm{m}$ for length, $3 \mathrm{ps}$ for time $t$, and hence c/400 (c = speed of light) for velocity $v$, furthermore $200 \mathrm{kV} / \mathrm{cm}$ for field $|\mathbf{E}|$, and $5 \cdot 10^{14} / \mathrm{cm}^{3} \approx 2 \cdot 10^{-5} \rho_{0}$ for the particle densities $\sigma$ and $\rho$, where $\rho_{0}$ is the particle density of the neutral $\mathrm{N}_{2}$ under normal conditions. The diffusion constant is $D=0.1$.

The minimal model applies to non-attaching gases like $\mathrm{N}_{2}$ or He. In attaching gases like $\mathrm{O}_{2}$, negative ions need to be included that can be formed by the attachment reaction: $\mathrm{e}^{-}+\mathrm{O}_{2} \longrightarrow \mathrm{O}_{2}^{-}$. In doped semiconductors, there are generally also three types of charged species: $(i)$ mobile electrons, $(i i)$ mobile electron holes and (iii) either immobile donors or immobile acceptors. In the reaction term (1.3), impact ionization by electron holes also needs to be taken into account. In direct band semiconductors such as, e.g., GaAs or GaN, or in $\mathrm{N}_{2} / \mathrm{O}_{2}$ mixtures, the possible importance of photoionization processes requires additional attention.

\subsection{Pattern formation in barrier discharges}

Before embarking into a more extensive discussion of streamer discharges, we briefly discuss barrier discharges with stationary (dc) or periodical (ac) drive as another manifestation of the ionizationfield-coupling.

In particular, planar layered systems with a sequence like $(i)$ transparent electrode, $(i i)$ gas discharge, (iii) resistive or dielectric layer (for dc or ac voltage, resp.), and ( $i v$ ) the second electrode are under continued careful experimental investigation $[7,8]$. The discharge and the linear layer are orders of mm thick, and the whole structure has a wide lateral aspect ratio. Looked at from above through the transparent electrode, these systems exhibit an extremely rich variety of effectively twodimensional patterns in the plane orthogonal to the layers. What is visible, is the light emitted from the ionized areas which is the sum of emissions over the whole height of the discharge layer.

The patterns resemble other effectively two-dimensional systems like Rayleigh-Bénard-convection or electroconvection in liquid crystals. The existence of a supercritical and a weakly subcritical bifurcation with associated amplitude patterns has been established [7], as well as a whole zoo of particle-like excitations with most striking interactions, replications and annihilations [7, 8].

However, a derivation of the patterns from gas discharge equations of type (1.5)-(1.7) has long been missing. An early attempt to predict the dc patterns [9] is based on the ad-hoc assumption of a locally bistable current-voltage-characteristics, which is common in semiconductor systems [4], but not applicable to discharges in nitrogen, as was discussed above. On the other hand, bistability of the discharge on a global level can result from the ionization-field-coupling. For appropriate boundary conditions, this instability can lead to the formation of a glow discharge with its characteristic space charges [1]. Pattern formation in the dc barrier discharge according to this mechanism is presently under investigation [10]. The starting point is a model like (1.5)-(1.7), where the mobility of the ions has to be included, but diffusion can be neglected.

In the case of ac barrier discharges [8], a field-ionization driven mechanism of pattern formation first was suggested in [11]. Indeed, recent numerical investigations [8] of a model of type (1.5)-(1.7) could verify this picture. 


\section{Pattern formation in Streamer Discharges}

\subsection{Basic observations}

From here on we focus on the transient process of dielectric breakdown by the streamer mechanism. Streamers occur, when a sufficiently high electric field is suddenly applied to an ionizable medium with low initial conductivity in a sufficiently long discharge gap. The effect was first studied by Raether [12], who experimentally observed a qualitative change of form and velocity of an impact ionization avalanche after a certain time of evolution. This avalanche-to-streamer-transition, he attributed to the space charges becoming important above a critical degree of ionization.

In terms of the minimal model, the avalanche is the regime of low particle densities. It amounts to neglecting the space charges $\rho-\sigma$ in (1.7). The field $\mathbf{E}=-\nabla \Phi$ then is completely determined by the boundary conditions and the Laplace equation $\nabla^{2} \Phi=0$. Eqs. (1.5) and (1.6) then are linear equations for the densities $\sigma$ and $\rho$, which have to be solved in the presence of a given external electric field. In the streamer regime, the contributions of the space charges $\rho-\sigma=\nabla \cdot \mathbf{E}(1.7)$ to the electric field become comparable to the external electric field. The ionization-field-coupling that then couples the three scalar fields $\sigma, \rho$ and $\Phi$, makes the full model (1.5)-(1.7) deeply nonlinear.

\subsection{Streamers of different flavors}

In $[13,6]$, the evolution of streamer discharges from initial ionization seeds was simulated for $\mathrm{N}_{2}$ under normal conditions between planar electrodes with a distance of $5 \mathrm{~mm}$ and a voltage of about $25 \mathrm{kV}$. The three dimensional problem was reduced to an effectively two dimensional one by the assumption of radial symmetry. Fig. 1 shows two time steps with $\Delta t=0.75 \mathrm{~ns}$ of the evolution of a negative streamer from Vitello et al. [6] - a negative or anode directed streamer propagates in the drift direction of the electrons, a positive or cathode directed streamer antiparallel to the electron drift. Each line in Fig. 1 denotes the increase of electron density by a factor of 10 . Densities from $10^{3}$ to $10^{14}$ free electrons $/ \mathrm{cm}^{3}$ can be seen. Thus the ionization increases by more than $10^{10}$ within a layer of a few $\mu$ m's thick.
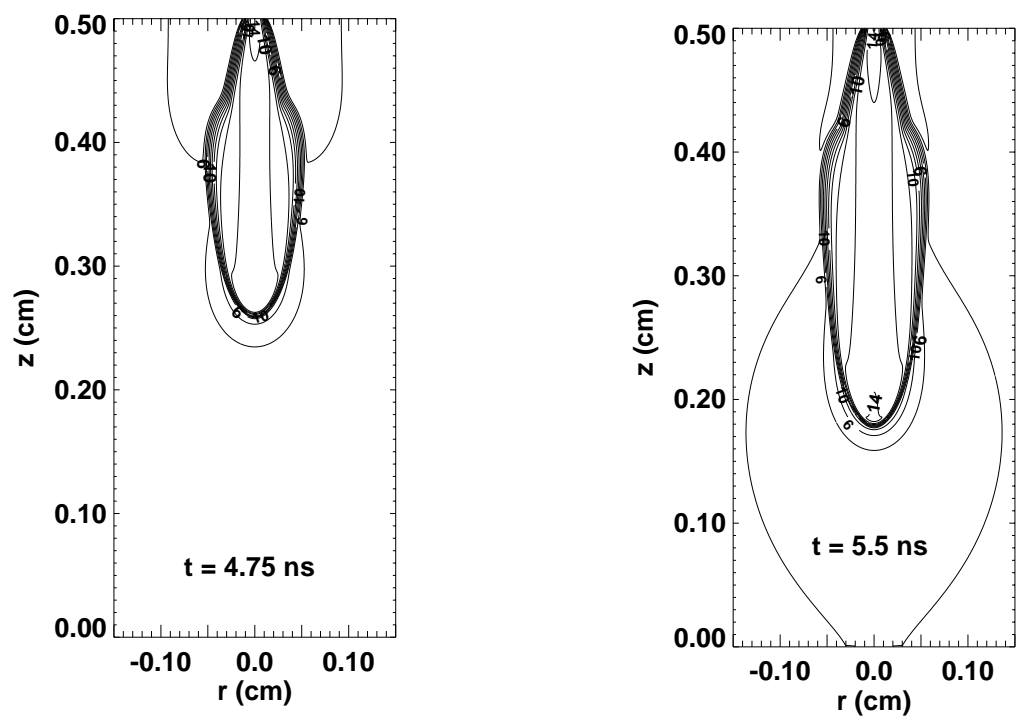

Figure 1: Figures from [6]: Two time steps of the evolution of a negative streamer into an area with very low background ionization [Courtesy of P.A. Vitello]. 
In dimensionless units, these simulations amount to numerical solutions of the model (1.5)-(1.7) with the dimensionless external field $E \approx 1 / 4$ and electrode distance $L \approx 250$. The dimensionless time difference is $\Delta t \approx 250$.

The interior of the streamer finger is an area of high ionization and low field, so the ionization reaction $\sigma f(|\mathbf{E}|)$ is suppressed, because $f(|\mathbf{E}|) \approx 0$. There is a well pronounced propagating front regime surrounding the finger, where reaction, drift, diffusion and electric screening take place. In the outer area, the reaction rate $\sigma f(|\mathbf{E}|)$ again is small, now because $\sigma$ is small. The two simulations $[13,6]$ differ by the properties of the outer area, which are determined by different initial conditions: In the earlier simulations of Dhali and Williams [13], the initial background ionization is $10^{8}-10^{11} / \mathrm{cm}^{3}$ $\left(=2 \cdot 10^{-7}-10^{-4}\right.$ in dimensionless units $)$, and the streamers propagate in an approximately symmetric manner in both directions of the electric field. In the simulations of Vitello et al. [6], the background ionization is $10^{3} / \mathrm{cm}^{3}\left(=2 \cdot 10^{-12}\right.$ in dimensionless units). This low background ionization makes the density gradients much steeper, too steep for the low spatial resolution of the earlier simulations [13]. The improved simulations [6] give a detailed picture of the propagation of negative streamers in low background ionization. The question whether positive streamers do propagate under these conditions, could numerically still not be resolved due to the much steeper gradients. Only the analysis [2] to be summarized below, could explain the nature of the gradients and the conditions of propagation of these fronts.

Thermal ionization of $\mathrm{N}_{2}$ at room temperature certainly can be neglected, since the Boltzmann factor is about $10^{-100}$. The background ionization in the atmosphere is caused by radioactivity and cosmic radiation. An average value is $10^{3} / \mathrm{cm}^{3}$ [14], so Vitello's simulations give a realistic picture of a negative streamer discharge in nitrogen - if the use of a continuum approximation for the initially very low density $10^{3} / \mathrm{cm}^{3}$ is appropriate. However, it seems reasonable to assume that during the evolution times of the simulations this small initial density is effectively negligible. Then these simulations show a picture of an essentially non-ionized outer area penetrated by the streamer. We will discuss the situation of streamers penetrating a non-ionized area in more detail below.

The simulations of Dhali and Williams [13] with the higher background ionization are realistic either for a pre-ionized gas or for semiconductor systems that actually can be thermally ionized at room temperature due to much lower ionization energies. A qualitative theory for such streamer discharges actually has been developed by D'yakonov and Kachorovskii [5]. Here the streamer velocity is essentially determined by the size of the "active" area around the streamer tip where the electric field is above the ionization threshold. The ionization wave then is a collective mode based on impact ionization and electric screening, which can become much faster than the speed of the local charge carriers. This is why the currents of the charge carriers are neglected in [5]. Therefore two basically different propagation modes should be distinguished: streamers in negligible background ionization and streamers in pre-ionized media.

Streamer-like impact ionization fronts propagating into a pre-ionized medium also are the mode of operation of semiconductor devices such as the microwave TRAPATT-diode [15] and the pulse sharpener diode [16]. In these layered semiconductor structures, depletion layers, small electrode distances or load resistors in the external circuit lead to additional stabilization or destabilization mechanisms $[17,18]$ of planar impact ionization fronts.

\subsection{Planar streamer fronts}

We now recall the basic analytical properties of planar streamers fronts [2], since this elucidates the dependence on the initial degree of ionization, and also sets the basis for the following analytical steps. Planar fronts that propagate according to the minimal model (1.5)-(1.7) with uniform velocity $v$ into an area with little ionization and time-independent homogeneous electric field $E^{+}$, solve the following set of nonlinear ordinary differential equations in a comoving coordinate frame $\xi=x-v t$ :

$$
\begin{array}{ll}
D \partial_{\xi}^{2} \sigma+(v+E) \partial_{\xi} \sigma+\sigma \partial_{\xi} E+\sigma f(|E|) & =0, \\
D \partial_{\xi} \sigma-v \partial_{\xi} E+\sigma E & =0 .
\end{array}
$$


In the area behind the front, the electric field is screened and the degree of ionization $\sigma^{-}$of the plasma is fully determined by the dynamics, not by any equilibrium process. The fronts can be constructed in the 3-dimensional phase space $\left(\sigma, \partial_{\xi} \sigma, E\right)$ as heteroclinic orbits between fixed points $\left(0,0, E^{+}\right)$and $\left(\sigma^{-}, 0,0\right)[2]$ - where actually the whole $E$ - and $\sigma$-axes are fixed point lines. In contrast to the more common fronts between a stable and a metastable state, the velocity $v$ here is not uniquely determined by the far field $E^{+}$. Rather a negative front can have any velocity $v \geq v^{*}$, where

$$
v^{*}=\left|E^{+}\right|+2 \sqrt{D f\left(\left|E^{+}\right|\right)},
$$

and a positive front can have any velocity $v \geq v^{\dagger}$, where

$$
v^{\dagger}=D \tilde{v}^{\dagger}\left(E^{+}\right)+O\left(D^{2}\right) .
$$

$\tilde{v}^{\dagger}\left(E^{+}\right)$is the nonlinear eigenvalue of a strongly heteroclinic orbit of eqs. (2.1)-(2.2) after rescaling with $D$ and then letting $D \rightarrow 0$, for details see [2]. Here negative/positive fronts are defined as carrying a negative/positive space charge in the front region and propagating parallel/antiparallel to the drift direction of electrons.

There is a continuous family of front solutions for given $E^{+}$, because the penetrated non-ionized state $\left(0,0, E^{+}\right)$is linearly unstable with respect to an infinitesimally small electron density $\sigma[2,19]$. If the electron density $\sigma(x, 0)$ initially vanishes as $e^{-\lambda x}$ for large $x$ with some $\lambda<\lambda_{\text {steep }}$, where

$$
\lambda_{\text {steep }}= \begin{cases}\sqrt{f\left(\left|E^{+}\right|\right) / D} & \text { neg. fronts, } \\ \left(v^{\dagger}+E^{+}-\sqrt{\left(v^{\dagger}+E^{+}\right)^{2}-4 D f\left(\left|E^{+}\right|\right)}\right) /(2 D) & \text { pos. fronts, }\end{cases}
$$

it actually will conserve its spatial decay rate in time and will approach an asymptotic front attractor with velocity $v=-E^{+}+D \lambda+f\left(\left|E^{+}\right|\right) / \lambda$ larger than $v^{*}$ or $v^{\dagger}$. If the spatial decay of $\sigma$ initially is slower than exponential, a planar front will accelerate. The slower the spatial decay, the higher will be the speed, and the more positive and negative fronts will resemble each other. This observation illustrates the similar propagation of positive and negative fronts in a system with high initial ionization [13].

On the other hand, sufficiently steep initial ionization profiles with

$$
\lim _{x \rightarrow \infty} \sigma(x, 0) e^{\lambda_{\text {steep }} x}=0
$$

for large times approach the "selected" velocity $v^{*}$ or $v^{\dagger}[2,19]$. In the case of negligible initial ionization, the slow propagation of positive fronts with velocity $v^{\dagger} \propto D$ and with a degree of ionization $\sigma^{-} \propto 1 / D$ behind the front [2] results from the balance of electron drift and diffusion, which leads to a singular dependence on $D$. This explains the numerical difficulties of the simulations of positive fronts [6]. However, the neglect of the ion motion then ceases to be a valid approximation. Hence we will not consider this solution any further. The negative front with sufficiently low background ionization, in contrast, propagates with the velocity $v^{*}(2.3)$ that is slightly higher than the drift velocity $\left|E^{+}\right|$ of an electron in an electric field $E^{+}$. The limit $D \rightarrow 0$ is smooth in this case [2].

\section{Streamers as a Laplacian growth problem}

In the remainder of the paper, we discuss the propagation of negative streamer fronts into an initial state with negligible ionization, and we will refer to the simulations [6] of Fig. 1.

For numerical convenience, the initial conditions of the simulations $[13,6]$ have a considerable ionization in some bounded part of space. Since such initial conditions are somewhat arbitrary and also might differ depending on prehistory, Vitello et al. [6] study in detail various initial conditions. It turns out that the late stage development of the propagating tip of the finger is always quite the same. This motivates the working hypothesis, that a certain late stage finger-shape is an attractor of the dynamics for a large class of initial conditions. 


\subsection{Pattern formation on the outer scale: Laplacian growth}

That finger-shaped moving interfaces can be attractors of the dynamics, is known from other pattern forming systems like viscous fingers or dendrites in solidification fronts [20]. This analogy can be made more precise [2]: The smallest length scale in Fig. 1 [6] is the width of the transition region between non-ionized and ionized region. If this length scale can be eliminated, the front can be modeled by an effectively infinitely thin interface on the outer scale.

Then the following reduced model emerges: The ionized area is conducting, so the field is low and the electric potential is

$$
\Phi \approx \text { const. } \quad \text { in the ionized region. }
$$

In the non-ionized area, there are no space charges, so that

$$
\nabla^{2} \Phi=0 \quad \text { in the non-ionized region. }
$$

If the electric field immediately in front of the ionization front has the time-independent value $\mathbf{E}^{+}$, then a planar front approaches a stationary state with velocity (2.3)

$$
v^{*}=\left|\mathbf{E}^{+}\right|+2 \sqrt{D f\left(\left|\mathbf{E}^{+}\right|\right)} \quad, \quad\left|\mathbf{E}^{+}\right|=\left.\hat{n} \cdot \nabla \Phi\right|_{+} .
$$

This is the velocity of the interface or moving boundary in the outer scale description. Here $\hat{n}$ is the orthonormal vector on the interface, and $\left.\nabla \Phi\right|_{+}$denotes the extrapolation of $\nabla \Phi$ from the non-ionized region onto the interface.

The moving boundary problem defined by (3.1)-(3.3) is the same as the lowest order approximation of viscous fingering in two dimensions, where an incompressible fluid of negligible viscosity penetrates an incompressible fluid of finite viscosity in a Hele-Shaw cell. The potential $\Phi$ then is replaced by pressure $p$.

It has been established in the gas discharge literature [21], that the model (3.1)-(3.3) with appropriate outer boundary conditions has uniformly translating paraboloid solutions. Mathematically speaking, these are equivalent to the Ivantsov paraboloids in dendritic growth [20].

\subsection{The need of a microscopic stabilization mechanism}

However, from studies of viscous fingering and dendritic growth, it is also known, that all paraboloid solutions are dynamically unstable under the dynamics of (3.1)-(3.3). In fact, an arbitrary initial condition generically will develop singularily curved interfaces within finite time. This fact is related to the short wave length instability of linear perturbations of planar fronts. A transversal Fourier perturbation $e^{i k x+s t}$ of a planar ionization front that propagates into a non-ionized area with field $E^{+}$, has the dispersion relation [22]

$$
s(k)=c\left(E^{+}\right) k \quad, \quad c\left(E^{+}\right)=\left.\frac{d v^{*}(E)}{d \ln |E|}\right|_{E^{+}} .
$$

The only difference to viscous fingering or dendritic growth is the weakly nonlinear dependence of $v^{*}$ on $E^{+}$for $D \neq 0$, which causes $v^{*}\left(E^{+}\right)$and $c\left(E^{+}\right)$to differ slightly.

In viscous fingering, e.g., an additional length scale not included in the model (3.1)-(3.3) is set by surface tension [20]. Inclusion of this length scale into the problem changes the dispersion relation to the form $s(k)=c k-d k^{3}$ and removes the short wave length instability. It also allows the accurate prediction of the actually realized finger-shape. The same holds for dendritic growth. Hence the question rises, which microscopic scale stabilizes the approximately uniformly translating shape of the streamer finger in Fig. 1. 


\subsection{Moving boundary approximations and pulled fronts}

The separation of inner and outer length scales that can be seen in the simulations, suggests the development of a moving boundary approximation from the streamer equations (1.5)-(1.7). Such an approximation substantiates the interface approximation above by matching different asymptotic expansions for the inner and outer scale. In particular, on the outer scale description of (3.1)-(3.2), it will lead, e.g., to a curvature correction to the interface velocity (3.3). Hence it seems appropriate to translate the derivation [23, 24] of a moving boundary approximation for PDE problems to the streamer equations in order to derive the corrections to (3.3) in a systematic manner.

A direct translation of these calculations to the streamer model, however, leads to a failure that can be placed in a broader context: the negative streamer front propagating into a non-ionized state belongs to the larger class of so-called pulled fronts [19]. For pulled fronts, the standard calculational methods break down. As is explained in more detail in [25], the calculations in [23, 24] assume implicitly that the interface can be parametrized by its geometric properties like curvature. The corrections to the interface velocity then are evaluated by solvability integrals, that arise from multiplication of the equations in first order perturbation theory of the form $\mathcal{L} \phi_{1}(\xi)=g(\xi)$ with a left zero mode of the linear operator $\mathcal{L}$. If the zero order solution about which one linearizes, is a pulled front, the solvability integrals diverge. This divergence is related to the divergence of the dynamically dominant area: while for fronts propagating into a metastable state or for so-called pushed fronts - an example of a pushed front is the positive streamer front propagating with velocity $v^{\dagger}(2.4)-$, the dynamics is determined in the interior nonlinear area of the front, for pulled fronts the dynamics is determined in the leading edge of the front, where linearization about the non-ionized state is a valid approximation. This area is half-infinite, and hence the spatial integral diverges.

This divergency can not be removed by an appropriate regularization, since it signals the breakdown of the underlying ansatz. The ansatz as in [23, 24] explicitly uses a separation of length scales between the width of the front and the outer scales, but it implicitly also assumes a separation of time scales between inner and outer dynamics. If the front is described by its geometric properties like curvature only, an adiabatic elimination of the inner time scale is assumed. This is not possible, if there is no inner time scale, and this is exactly the case for pulled fronts. This lack of an inner time scale is illustrated in particular by the relaxation of a negative planar streamer front with sufficiently steep initial conditions (2.6) towards its asymptotic velocity $v^{*}(2.3)$. It is asymptotically for large $t$

$$
v(t)=\left|E^{+}\right|+\sqrt{D f\left(E^{+}\right)}\left[2-\frac{3}{2 \tau}+\frac{3 \sqrt{\pi}}{2 \tau^{3 / 2}}+\mathcal{O}\left(\frac{1}{\tau^{2}}\right)\right] \quad, \quad \tau=f\left(E^{+}\right) t .
$$

This algebraic relaxation that is universal for all pulled fronts [19], has up to order $1 / t$ no intrinsic time scale, in contrast to the more common pushed or bistable fronts that relax exponentially fast in time.

\subsection{Possible stabilization mechanisms}

The problems in the formal derivation of a moving boundary approximation from the streamer equations (1.5)-(1.7) are due to the leading edge dominated dynamics of pulled fronts. The exponentially decaying leading edge is generated by diffusion: for $D=0$, the ionization front in a field $E^{+}$becomes a shock front propagating with the drift velocity $v=\left|E^{+}\right|$. Inspection of (3.5) shows, that for $D=0$ also the algebraic relaxation is removed. Still there is a length scale in the problem, which is not yet incorporated in the lowest order interface approximation (3.1)-(3.3), namely the electric screening length $\ell_{\alpha}=1 / \alpha\left(E^{+}\right)=\left|E^{+}\right| / f\left(E^{+}\right)$. It is related to the Maxwell relaxation time $t_{M}=1 / f\left(E^{+}\right)$.

The planar uniformly translating negative streamer shock front for $D=0$ can be solved analytically: assume that in the comoving frame $\xi=x-v t$, the position of the ionization shock is $\xi=0$. For $\xi \geq 0$, there are no charge carriers, and the electric field has the constant value $E^{+}<0$. For $\xi<0$, one can 
express electron and ion densities $\sigma$ and $\rho$ and coordinate $\xi$ as a function of $E$ as [2]

$$
\rho[E]=\int_{|E|}^{v} d \epsilon \alpha(\epsilon) \quad, \quad \sigma[E]=\frac{v \rho[E]}{v+E} \quad, \quad \xi=\int_{|E(-\xi)|}^{v} d \epsilon \frac{v-\epsilon}{\epsilon \rho[\epsilon]} \quad, \quad v=\left|E^{+}\right| .
$$

At $\xi=0, \sigma$ jumps to the finite value $\sigma\left(0^{-}\right)=f\left(E^{+}\right)=\left|E^{+}\right| \alpha\left(E^{+}\right)$, and then decreases for $\xi \rightarrow-\infty$ to the neutral plasma density $\sigma(-\infty)=\int_{0}^{v} d \epsilon \alpha(\epsilon)$. Also the derivatives of $\rho$ and $E$ are discontinuous at $\xi=0$.

Can the additional length scale $\ell_{\alpha}$ lead to a stabilization of the short wave length instability of (3.4)? Preliminary results [22] do show the appearance of the length scale $\alpha\left(E^{+}\right)$in the dispersion relation

$$
s(k)=\left\{\begin{array}{ll}
\left|E^{+}\right| k & \text { for } k \ll 1 \\
\left|E^{+}\right| \alpha\left(E^{+}\right) / 2 & \text { for } k \gg 1
\end{array},\right.
$$

but a short wave length instability still stays present in the dispersion relation of linear transversal perturbations of a planar front.

At present, we investigate two working hypotheses: either diffusion $D$ needs to be included to remove the short wave length instability, or the instability is removed from a sufficiently curved drift front with $D=0$ by electrostatic effects [22]. This would then allow an outer scale analysis of improved interface equations (3.1)-(3.3) and a prediction of the asymptotic form of a streamer finger.

\section{Summary and outlook}

We have discussed two pattern forming systems in electric discharges, namely barrier and streamer discharges. In barrier discharges, one can realize, e.g., stationary patterns in layered systems with wide lateral aspect ratios, which show similarities to, e.g., Rayleigh-Benard-convection. Streamer discharges are transient finger-shaped ionized patterns penetrating an area with very low or vanishing ionization, similar to viscous fingers or dendrites.

Both barrier and streamer discharges are characterized by low ionization and current densities. Hence the patterns in simple media like pure nitrogen should be described by the minimal discharge model (1.5)-(1.7). This model is a reaction-drift-diffusion model for two charged species coupled to the electric field. The field determines reaction and drift, while the space charges modify the field. This leads to nonlinear dynamics with a ionization-field-coupling, if the particle densities and hence the space charges are sufficiently large.

In particular, we discussed the penetration of a non-ionized area with high electric field by an ionized negative streamer finger. We identified this problem as a Laplacian growth problem quite comparable to viscous fingering or dendritic growth. For a complete moving interface approximation of streamer propagation, a correction to the velocity due to curvature or relaxation is still missing. This is due to the particular properties of negative streamer fronts that belong to the larger class of pulled fronts. For pulled fronts, standard moving boundary approximations fail, because there is no separation of inner and outer time scales. We presented first steps towards the derivation of a different approximation.

\section{ACKNOWLEDGEMENT}

We thank P. Rodin for productive discussions about streamer propagation in pre-ionized media, and for advice and references on streamer discharges in semiconductor devices. Many aspects of the nonlinear analysis presented in this review were shaped during previous collaborations of U.E. with W. van Saarloos. We thank P.A. Vitello for the permission to reprint Fig. 1. U.E. was supported by the Dutch Science Foundation NWO, and M.A. by the EU-network "Patterns, Noise, and Chaos". 


\section{References}

1. Y.P. Raizer: Gas Discharge Physics (Springer, Berlin 1991)

2. U. Ebert, W. van Saarloos, C. Caroli: Phys. Rev. Lett. 77, 4178 (1996); and Phys. Rev. E 55, $1530(1997)$

3. J. Meunier et al.: J. Appl. Phys. 78, 731 (1995); C. Punset et al.: J. Appl. Phys. 83, 1884 (1998)

4. E. Schöll: Nonequilibrium Phase Transitions in Semiconductors (Springer, Berlin 1987)

5. M.I. D'yakonov, V.Y. Kachorovskii: Sov. Phys. JETP 67, 1049 (1988); and Sov. Phys. JETP 68, 1070 (1989)

6. P.A. Vitello, B.M. Penetrante, J.N. Bardsley: Phys. Rev. E 49, 5574 (1994)

7. Yu. Astrov, E. Ammelt, S. Teperick, H.-G. Purwins: Phys. Lett. A 211, 184 (1996); Yu. Astrov et al.: Phys. Rev. Lett. 78, 3129 (1997), Phys. Rev. Lett. 79, 2983 (1997), Phys. Rev. Lett. 80, 5341 (1998); E. Ammelt et al.: Phys. Rev. E 55, 6731 (1997), Phys. Rev. E 58, 7109 (1998); C. Strümpel et al.: Phys. Rev. E 61, 4899 (2000)

8. E. Ammelt, D. Schweng, H.-G. Purwins: Phys. Lett. A 179, 348 (1993); I. Brauer et al.: J. Appl. Phys. 85, 7569 (1999), Phys. Rev. Lett. 84, 4104 (2000); I. Müller et al.: Phys. Rev. Lett. 82, 3428 (1999), IEEE Trans. Plasma Science 27, 20 (1999)

9. C. Radehaus et al.: Phys. Rev. A 45, 2546 (1992)

10. Yu. Astrov: [private communication]; D. Sijacic, U. Ebert: [in preparation]

11. W. Breazeal, K.M. Flynn, E.G. Gwinn, Phys. Rev. E 52, 1503 (1995)

12. H. Raether: Z. Phys. 112, 464 (1939) (in German); and Ergebnisse der exakten Naturwissenschaften 22, 73 (1947) (in German)

13. S.K. Dhali, P.F. Williams: Phys. Rev. A 31, 1219 (1985); and J. Appl. Phys. 62, 4696 (1987)

14. A.M. Howatson: An Introduction to Gas Discharges, 2nd edn. (Pergamon, Oxford 1976)

15. B.C. Deloach, D.L. Scharfetter: IEEE Trans. El. Dev. ED-20, 9 (1970)

16. I.V. Grekhov, A.F. Kardo-Sysoev: Sov. Tech. Phys. Lett. 5, 395 (1979)

17. S.N. Vainstein, Yu.V. Zilyaev, M.E. Levinstein: Sov. Tech. Phys. Lett. 14, 920 (1998); Sov. Phys. Semicond. 22. 717 (1988)

18. A.M. Minarsky, P.B. Rodin: Semiconductors 31, 356 (1997); Sol. St. Electron., 41, 813 (1997) 
19. W. van Saarloos: Phys. Rev. A 39, 6367 (1989); U. Ebert, W. van Saarloos: Phys. Rev. Lett. 80, 1650 (1998); and Physica D 146, 1 (2000)

20. For a review and a collection of older relevant articles see P. Pelcé: Dynamics of Curved Fronts (Academic Press, San Diego 1988)

21. This can be concluded from a remark in [5], referring to E.D. Lozanskii, O.B. Firsov: Spark Theory (Atomizdat, 1975) (in Russian). From the remark, the solution can be reconstructed using the calculation of the field outside any conducting ellipsoid, see, e.g., L.D. Landau, E.M. Lifshitz, paragraph 4 of vol. VIII: Electrodynamics of Continuous Media (Pergamon, Oxford 1960)

22. M. Arrayás, U. Ebert [in preparation]

23. P. Fife: Dynamics of Internal Layers and Diffusive Interfaces (SIAM, Philadelphia 1988)

24. A. Karma, W.J. Rappel: Phys. Rev. E 53, R3017 (1996); and Phys. Rev. E 57, 4323 (1998)

25. U. Ebert, W. van Saarloos: Phys. Rep. 337, 139 (2000) 